\title{
Pathological Forms of Narcissism and Perceived Stress During the Transition to the University: The Mediating Role of Humor Styles
}

\author{
Avi Besser \\ Sapir Academic College, Israel \\ Virgil Zeigler-Hill \\ Oakland University
}

The way individuals use humor is likely to be influenced by how they feel about themselves. The goal of the present studies was to examine the association between the pathological forms of narcissism (grandiose and vulnerable narcissism) and humor styles in Jewish Israeli undergraduate samples as they made the adjustment to being university students. Study 1 $(\mathrm{N}=187)$ found that grandiose narcissism was positively associated with adaptive humor, whereas vulnerable narcissism was negatively associated with adaptive humor and positively associated with maladaptive humor. Study $2(\mathrm{~N}=251)$ found that humor styles mediated the associations between the pathological forms of narcissism and perceived stress. There was no evidence of any moderating effect of humor styles on the relationship between the pathological forms of narcissism and perceived stress. These findings are discussed in terms of the role that humor may play in explaining the association between the pathological forms of narcissism and perceived stress.

Keywords: narcissism, grandiose, vulnerable, humor, stress

This article was published Online First July 18, 2011.

Avi Besser, Department of Behavioral Sciences and Center for Research in Personality, Life Transitions, and Stressful Life Events, Sapir Academic College; Virgil Zeigler-Hill, Department of Psychology, Oakland University.

We would like to acknowledge research assistances of Sapir Academic College, Israel, and of Ben Gurion University of the Negev Eilat Campus, Israel, for their valuable assistance in the data collection. Grateful thanks are extended to all of the participants in this study. Finally, We thank the anonymous reviewers for their constructive suggestions and comments on a draft of the paper.

Correspondence concerning this article should be addressed to Avi Besser, Department of Behavioral Sciences, Sapir Academic College, D. N. Hof Ashkelon 79165, Israel. E-mail: besser@mail.sapir.ac.il 
Humor has been conceptualized in various ways. The classic psychodynamic model of humor developed by Freud $(1905 / 1960,1928)$ suggested that individuals often use humor to defend themselves against feelings of anxiety or to express unconscious desires (e.g., aggression), whereas later theorists suggested that humor serves an array of functions such as perspective-taking (e.g., Allport, 1961; Maslow, 1954; see Martin, Puhlik-Doris, Larsen, Gray, \& Weir, 2003, for a review). An important innovation in research concerning humor has been the recent development of a two-dimensional framework for understanding the interpersonal nature of humor (Martin et al., 2003). Humor is primarily a social phenomenon that serves a variety of interpersonal functions including social control, status maintenance, and group cohesion (Martin, 2007). The framework developed by Martin and his colleagues (2003) suggests that there are two underlying dimensions that reflect both the interpersonal nature of humor (i.e., injurious or benign) as well as the target of enhancement (i.e., the self or relationships with others). The combination of these two dimensions results in four distinct humor styles that are referred to as affiliative humor (i.e., benign humor that is used to enhance relationships with others such as telling jokes or engaging in friendly banter), self-enhancing humor (i.e., benign humor that is used to enhance the self through means such as finding amusement even during stressful situations), aggressive humor (i.e., injurious humor that is used to enhance the self such as ridiculing or teasing others to put them down), and self-defeating humor (i.e., injurious humor that is used to enhance relationships with others through actions such as belittling oneself).

The underlying dimensions of humor and the four humor styles that emerge from their combination are depicted in Figure 1. The relatively

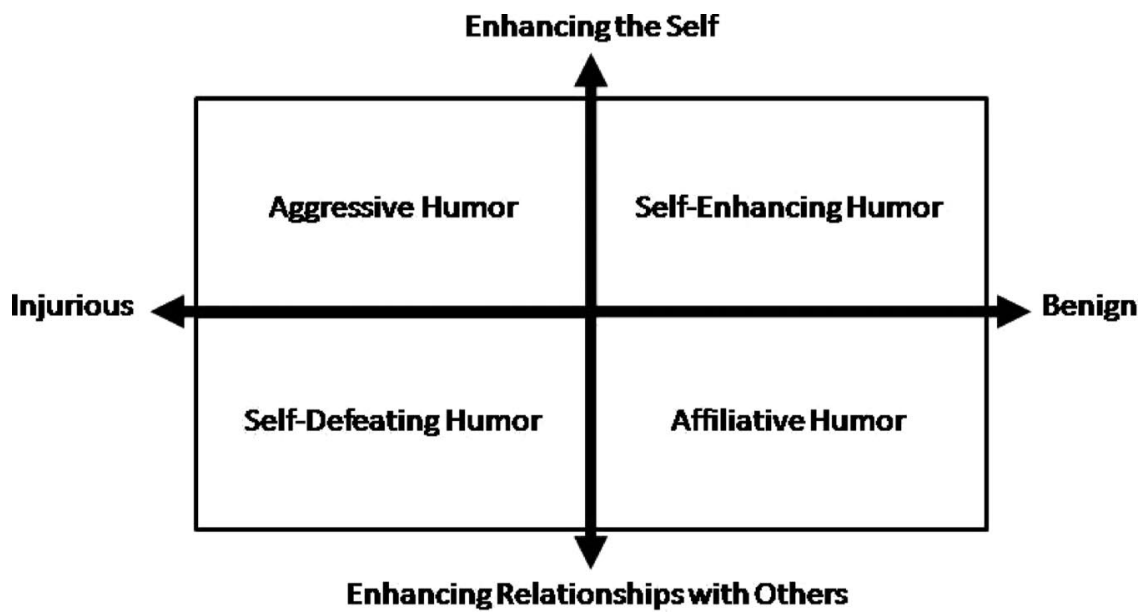

Figure 1. The four humor styles described by Martin et al. (2003). 
benign (or adaptive) styles of humor (i.e., affiliative and self-enhancing) have been found to be associated with personality features such as high levels of extraversion, openness, and self-esteem. In contrast, the more injurious (or maladaptive) styles of humor (i.e., self-defeating and aggressive) have been found to be associated with high levels of neuroticism, psychopathy, and Machiavellianism, as well as low levels of agreeableness and conscientiousness (Galloway, 2010; Martin et al., 2003; Vernon, Martin, Schermer, \& Mackie, 2008; Veselka, Schermer, Martin, \& Vernon, 2010; see Martin, 2007, for a review). The differentiation between adaptive and maladaptive styles of humor is largely consistent with previous views of humor that recognized the fact that humor can be used in different ways and serve different purposes (e.g., Freud, 1905/1960).

One factor that may influence how individuals use humor is how they feel about themselves. For example, self-esteem has been found to be positively correlated with the adaptive styles of humor and negatively correlated with the maladaptive styles (Galloway, 2010). That is, individuals with high self-esteem tend to use benign forms of humor that serve affiliative or self-enhancing goals rather than forms of humor that are injurious. The link between high levels of self-esteem and humor styles is particularly interesting given recent research showing that high self-esteem is associated with interpersonal styles characterized by a blend of dominance and affiliation (e.g., Zeigler-Hill, 2010; Zeigler-Hill, Clark, \& Beckman, in press). Consistent with these self-esteem findings, narcissism - which refers to grandiose and inflated views of the self- has been found to be associated with the adaptive forms of humor but not the maladaptive forms (Veselka et al., 2010). Narcissism is characterized by an assured-dominant interpersonal style that focuses on interpersonal control and social dominance (e.g., Gurtman, 1992; Ruiz, Smith, \& Rhodewalt, 2001). According to Foa and Foa (1974), the interpersonal style of narcissists is characterized by a pattern of social exchange in which both love and status are granted to the self but only love is granted to others while status is withheld from them. This suggests that narcissists may use affiliative and self-enhancing humor styles to foster positive relationships with others which may indirectly serve their selfesteem regulation goals (i.e., they may feel better about themselves when they receive the respect and admiration of others; Morf \& Rhodewalt, 2001).

Interest in narcissism spans both clinical and social-personality psychology but it has been difficult to integrate these bodies of literature because of differences in their conceptualization and measurement of narcissism (Cain, Pincus, \& Ansell, 2008; Miller \& Campbell, 2008; Pincus, Ansell, Pimentel, Cain, Wright, \& Levy, 2009). Clinical psychologists generally think of narcissism in terms of the personality disorder that is often believed to be associated with arrogant or haughty behaviors, feelings of entitlement, a lack of empathy, and a willingness to exploit other individuals (American Psy- 
chiatric Association, 2000). This form of narcissism is characterized by emotional instability such that these individuals tend to experience negative emotional states. In contrast, social-personality psychologists usually focus on subclinical levels of narcissism and conceptualize it as a normally distributed personality feature that has elements of emotional resilience and extraversion because it blends the relatively adaptive properties of narcissism (e.g., leadership and authority) with those that are maladaptive (e.g., exploitation and entitlement; see Miller \& Campbell, 2008, or Pincus \& Lukowitsky, 2010, for extended discussions). These differences result in clinical psychologists thinking of narcissism as a more pathological construct than the way in which it is typically viewed by social-personality psychologists. Consequently, new assessment tools such as the Pathological Narcissism Inventory (PNI; Pincus et al., 2009) have been developed to assess the more pathological form of narcissism that is not adequately captured by commonly used measures such as the Narcissistic Personality Inventory (NPI; Raskin \& Hall, 1979, 1981). It is important to note that the form of narcissism that has previously been examined in conjunction with humor has been the relatively healthy and adaptive form of narcissism that is assessed by the NPI (e.g., Veselka et al., 2010).

Pathological narcissism is a complex and heterogeneous construct that consists of both a grandiose and a vulnerable form (see Pincus \& Lukowitsky, 2010, for a review). The grandiose form of pathological narcissism is the most easily recognized because it is characterized by maladaptive selfenhancement strategies such as holding an overly positive self-image, exploiting others, and engaging in exhibitionistic behaviors. In contrast, the vulnerable form of pathological narcissism is characterized by self and emotional dysregulation that includes self-criticism, negative affective experiences, and social problems (e.g., interpersonal sensitivity, social withdrawal). The existence of grandiose and vulnerable forms of pathological narcissism has been supported by a number of studies (see Pincus \& Lukowitsky, 2010, for a review).

\section{OVERVIEW AND PREDICTIONS}

The goal of the present studies was to expand what is currently known about the connection between narcissism and stress by examining the role that humor plays in their association. For example, narcissism has been shown to be associated with stress (Edelstein, Yim, \& Quas, 2010; Kelsey, Ornduff, McCann, \& Reiff, 2001; Sommer, Kirkland, Newman, Estrella, \& Andreassi, 2009) but the present studies have the potential to improve our understanding of this link by determining how the use of 
humor influences the level of perceived stress reported by narcissistic individuals. Study 1 focused on establishing the connection between the pathological forms of narcissism and humor styles. We expected grandiose narcissism to be associated with the adaptive forms of humor but not with the maladaptive forms of humor. The underlying rationale was that individuals with high levels of grandiose narcissism may use adaptive forms of humor in an attempt to gain the approval of others because this is important for their ability to regulate their feelings of self-worth. This prediction is consistent with the results of Veselka et al. (2010) that found that the form of narcissism captured by the NPI was associated with the affiliative and self-enhancing humor styles but not the aggressive or self-defeating humor styles.

Our predictions for vulnerable narcissism were less certain than our predictions for grandiose narcissism because less research has focused on this form of pathological narcissism. Our tentative expectation was that vulnerable narcissism would be associated with the maladaptive forms of humor. Our basis for this prediction was that vulnerable narcissism is characterized by a tendency to engage in self-criticism and to experience intense anger when their feelings of entitlement are violated (Besser \& Priel, 2009, 2010; Cooper, 1998; Gabbard, 1989, 1998; Gersten, 1991; Wink, 1991). As a result, it seemed reasonable to expect that individuals with high levels of vulnerable narcissism may report using humor styles that were self-defeating and aggressive.

The purpose of Study 2 was to examine whether humor styles mediated the association between the pathological forms of narcissism and perceived stress during the transition to life as a university student. Our prediction was that the humor styles that individuals adopt may provide at least a partial explanation for their perceptions of stress (e.g., Abel, 1998, 2002; Kuiper \& Martin, 1998; Labott, Ahleman, Wolever, \& Martin, 1990; Martin, Kuiper, Olinger, \& Dance, 1993). This is important because individuals with high levels of the pathological forms of narcissism have been found to be highly reactive to negative events (e.g., Besser \& Priel, 2010; Besser \& Zeigler-Hill, 2010).

\section{METHOD}

\section{Participants and Procedure}

Participants were 187 undergraduates (56 men and 131 women) who took part during the first week of their first semester at a university or a college in the southern region of Israel. Participants were recruited for a study 
concerning the transition to the university using an Internet-based campus message board after they had received the syllabi for their courses and had the opportunity to consider the academic demands being placed on them. All of the participants were young Jewish Israeli adults who had already served 2-4 years of mandatory service in the Israeli army. It is important to note that Israeli students are generally older than most Western students (i.e., the mean age of our participants was 23.61 years [range 20-30; $S D=3.08$ ]).

Participation in the study was voluntary and participants were not paid or compensated for their participation except for those who were enrolled in psychology courses and participated in return for partial fulfillment of a research participation requirement. Although participants were reminded that they could discontinue their participation in the study at any time, none of the participants elected to do so. Participants were asked to provide written informed consent after the procedures had been fully explained. Participants were then asked to complete measures of pathological narcissism and humor styles-as well as other measures that are not relevant to the present study-during a 45-min laboratory session. All of the questionnaires used in the present study were administered in Hebrew after being translated from the original English versions using the backtranslation method. Potential order effects were controlled by presenting the questionnaires in a randomized order. Upon completion of the study, the participants were provided a written debriefing.

\section{Measures}

\section{Pathological Narcissism}

The PNI (Pincus et al., 2009) was used to assess the grandiose and vulnerable aspects of pathological narcissism. The PNI is a 52-item measure for which responses were made on scales ranging from 0 (not at all like me) to 5 (very much like me). This instrument captures seven dimensions of pathological narcissism: contingent self-esteem (e.g., "It's hard for me to feel good about myself unless I know other people like me"), exploitative tendencies (e.g., "I can make anyone believe anything I want them to"), self-sacrificing self-enhancement (e.g., "I try to show what a good person I am through my sacrifices"), hiding of the self (e.g., "When others get a glimpse of my needs, I feel anxious and ashamed"), grandiose fantasy (e.g., "I often fantasize about being recognized for my accomplishments"), devaluing (e.g., "When others don't meet my expectations, I often feel ashamed about what I wanted"), and entitlement rage (e.g., "It irritates me when people don't notice how good a person I am"). 
As outlined in recent studies (Tritt, Ryder, Ring, \& Pincus, 2010; Wright, Lukowitsky, Pincus, \& Conroy, 2010), these seven dimensions load onto two higher-order factors referred to as grandiose narcissism (exploitative, self-sacrificing self-enhancement, and grandiose fantasy) and vulnerable narcissism (contingent self-esteem, hiding the self, entitlement rage, and devaluing). Initial information concerning the reliability and validity of the PNI has shown that it is correlated in the expected direction with other measures of narcissism (e.g., NPI) as well as related constructs such as self-esteem level, interpersonal style, clinical outcomes, and contingent self-esteem (e.g., Pincus et al., 2009; Zeigler-Hill, Clark, \& Pickard, 2008). The internal consistencies of the PNI grandiosity and vulnerability subscales were .88 and .85 , respectively.

\section{Humor Styles}

The Humor Styles Questionnaire (Martin et al., 2003) was used to assess adaptive and maladaptive humor styles. It is a 32-item measure that consists of four subscales that assess the following styles of humor: affiliative (e.g., "I laugh and joke a lot with my friends"; $\alpha=.73$ ), self-enhancing (e.g., "My humorous outlook on life keeps me from getting overly upset or depressed about things"; $\alpha=.84$ ), aggressive (e.g., "If someone makes a mistake, I will often tease them about it"; $\alpha=$ .71), and self-defeating (e.g., "I let people laugh at me or make fun at my expense more than I should"; $\alpha=.81$ ). Responses were made on scales ranging from 1 (totally disagree) to 7 (totally agree). Martin et al. (2003) demonstrated good reliability and validity for this measure.

\section{RESULTS}

\section{Data Analytic Strategy}

Our analyses focused on the proposed associations between the pathological forms of narcissism (i.e., grandiose and vulnerable) and the humor styles (i.e., adaptive and maladaptive) using a Structural Equation Modeling (SEM; Hoyle \& Smith, 1994) strategy that assessed measurement errors in the dependent and independent variables. All analyses were conducted with AMOS (Version 18; Arbuckle, 2009) using the maximum-likelihood method in which we specified adaptive and maladaptive humor (each with two indicators as previously confirmed and demonstrated in samples of community adults using CFA strategy [Besser, 
Luyten, \& Blatt, in press; Besser, Luyten, \& Mayes, submitted for publication] as well as in samples of university students [Zeigler-Hill \& Besser, 2011] $)^{1}$. The following fit indices were used: the $\chi^{2} / d f$ ratio, the Root Mean Square Error of Approximation (RMSEA), the Comparative Fit Index (CFI), and the Non-Normed Fit Index (NNFI). A model in which/ $\chi^{2} / d f$ was $\leq 3, C F I$ and NNFI were greater than .90 , and the RMSEA index was between .00 and .06 with confidence intervals between .00 and .08 (Hu \& Bentler, 1999) was considered acceptable. These moderately stringent acceptance criteria clearly reject inadequate or poorly specified models while accepting models that meet real-world criteria for reasonable fit and representation of the data (Kelloway, 1998). The zero-order correlations between the study variables are displayed in Table 1.

\section{Pathological Forms of Narcissism and Humor Styles}

We ran an SEM model that included the simultaneous association of the grandiose and vulnerable forms of pathological narcissism with adaptive and maladaptive humor styles which is depicted in Figure 2. This model fit the observed data very well: $\chi^{2}(5)=6.36, p>.27, \chi^{2} / d f=1.27, \mathrm{NNFI}=.97$, $\mathrm{CFI}=.99, \mathrm{RMSEA}=.04$ [90\% CI $0.000,0.05]$. This model indicated that grandiose narcissism was positively associated with adaptive humor $(\beta=$ $.46, t=3.48, p<.001)$ and vulnerable narcissism was negatively associated with adaptive humor $(\beta=-.40, t=3.14, p<.01)$. Grandiose narcissism was not associated with maladaptive humor $(\beta=.13, t=1.06, n s)$ but vulnerable narcissism was positively associated with this form of humor $(\beta=$ $.31, t=2.33, p<.02)$.

\section{DISCUSSION}

The results of Study 1 provided partial support for our predictions. More specifically, grandiose narcissism was associated with the adaptive

${ }^{1}$ It is important to note that we conducted Confirmatory Factor Analysis- Scale structure (CFA; Anderson \& Gerbing, 1988) to investigate the fit of a model with two correlated latent factors (i.e., adaptive humor and maladaptive humor) with each defined by two observed variables that had an acceptable fit. All of the factor indicators and path loadings were substantial and statistically significant in the expected directions. Moreover, to exclude another model, we also examined whether we could rule out the possibility of subsuming adaptive and maladaptive humor into one overarching "humor styles" construct. This one latent construct model did not fit the data well and had a significantly worse fit compared to the model with two latent constructs. These results emerged in both of the present studies and are consistent with previous research. 
Table 1. Descriptive Statistics and Zero-Order Correlations

\begin{tabular}{|c|c|c|c|c|c|c|}
\hline & 1 & 2 & 3 & 4 & 5 & 6 \\
\hline \multicolumn{7}{|c|}{ Study 1} \\
\hline 1. Grandiose narcissism & - & & & & & \\
\hline 2. Vulnerable narcissism & $.61^{\text {**** }}$ & - & & & & \\
\hline 3. Affiliative humor & $.15^{*}$ & -.10 & - & & & \\
\hline 4. Self-enhancing humor & $.15^{*}$ & -.07 & $.45^{* * * *}$ & - & & \\
\hline 5. Aggressive humor & $.23^{* * *}$ & $.22^{* *}$ & .12 & $.15^{*}$ & - & \\
\hline 6. Self-defeating humor & $.14^{*}$ & $24^{* * * *}$ & .02 & $.17^{*}$ & $.35^{* * *}$ & - \\
\hline$M$ & 2.62 & 1.83 & 5.29 & 4.31 & 3.21 & 2.67 \\
\hline \multirow[t]{2}{*}{$S D$} & 0.75 & 0.75 & 0.84 & 1.23 & 0.88 & 1.03 \\
\hline & 1 & 2 & 3 & 4 & 6 & 7 \\
\hline
\end{tabular}

Study 2

1. Grandiose narcissism

2. Vulnerable narcissism

3. Affiliative humor

4. Self-enhancing humor

5. Aggressive humor

6. Self-defeating humor

7. Perceived stress

$.60^{* * *}$

$.60^{* * * *}-.13^{* * *}$

$.14^{*}-.10$

$.21^{* * * *} \quad .22^{* * *}$

$.20^{* * * *}$

$.15^{*}$

$.22^{\text {*** }} .27^{* * * * *}$

$.37^{* * * *}$

$\overline{-}^{\text {**** }}$
.11
.08
-.12

1.82

5.36

0.78

0.88

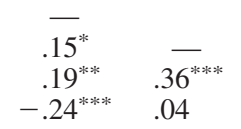

$\begin{array}{ll}S D & 0.75\end{array}$

4.29

3.20

1.22

$p<.05 .{ }^{* * *} p<.01 .{ }^{* * * *} p<.001$.

humor styles but not with the maladaptive humor styles. This is consistent with previous research showing a similar pattern for the form of narcissism captured by the NPI (Veselka et al., 2010). The positive association between vulnerable narcissism and maladaptive humor was consistent with our predictions but we did not expect the negative association between vulnerable narcissism and adaptive humor that emerged. The tendency for individuals with high levels of vulnerable narcissism to use maladaptive humor and to refrain from using adaptive humor may provide at least a partial explanation for their interpersonal difficulties that have been observed in previous studies (e.g., interpersonal distress, social avoidance; Dickinson \& Pincus, 2003).

\section{STUDY 2: PATHOLOGICAL FORMS OF NARCISSISM, HUMOR STYLES, AND PERCEIVED STRESS}

The purpose of Study 2 was to examine whether adaptive and maladaptive humor styles mediated the association between the forms of pathological narcissism and perceived stress during the transition to life as a university student. We chose to examine the transition to higher education because this is often a period that creates considerable stress in the lives of these individuals. Stress generally refers to the perception that there is a discrepancy between environ- 


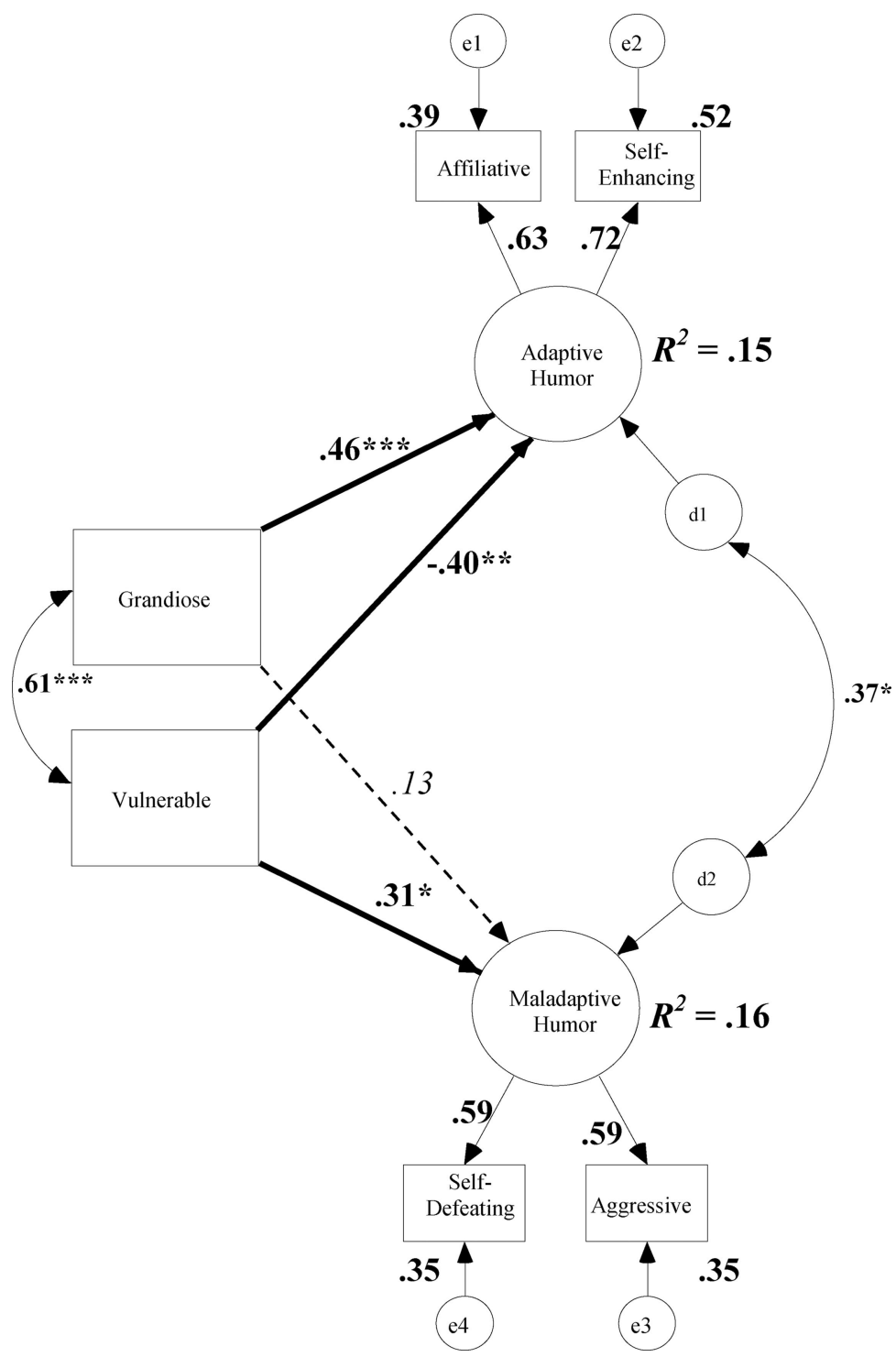

Figure 2. Study 1: Model of the associations between the pathological forms of narcissism and humor styles. Note. Rectangles indicate measured variables and the large circles represent latent constructs. Small circles reflect residuals (e). Bold numbers above or near endogenous variables represent the amount of variance explained $\left(R^{2}\right)$. Bidirectional arrow depicts covariance and unidirectional arrows depict hypothesized directional links. Standardized maximum likelihood parameters are used. Bold estimates are statistically significant. $N=187$; ${ }^{* *} p<.01$. **** $p<.001$. 
mental demands (stressors) and individual capacities to fulfill or cope with these demands (e.g., Lazarus \& Folkman, 1984; Malach-Pines \& Keinan, 2007; Vermunt \& Steensman, 2005). First-year college students are a group that is particularly prone to stress because of the transitional nature of college life (D’Zurilla \& Sheedy, 1991; Towbes \& Cohen, 1996). These students face an array of stressors that are related to these new academic demands that include adapting to new learning environments and methods of instruction, receiving less individual support from instructors, competing for grades, trying to effectively manage more demands for their time, and engaging in the self-regulation that is necessary to develop their skills (e.g., Fram \& Bonvillian, 2001; Macan, Shahani, Dipboye, \& Phillips, 1990; Trueman \& Hartley, 1996). It is not uncommon for students to struggle during this transition period because they may not be fully prepared for higher education (e.g., lack appropriate study skills; Haggis \& Pouget, 2002) and may feel alienated from their social support networks (Walker, Matthew, \& Black, 2004). Although Israeli college students are often somewhat older and more experienced than students in many other countries, they still tend to experience considerable stress during this transition period (e.g., Shirom, 1986).

Humor has been shown to protect individuals from the negative effects of stress but the precise mechanism by which this buffering effect occurs is poorly understood. It is possible that the use of humor leads to more positive or realistic appraisals (e.g., Abel, 1998, 2002; Kuiper \& Martin, 1998; Labott et al., 1990). The benefits of humor may also be because of the ability of humor to cause a cognitive-affective shift that restructures how the individual perceives the situation in a way that reduces the negative affective consequences of the stressor (Martin et al., 1993). Another way that humor may buffer individuals from stress is its ability to create psychological "distance" between oneself and the negative experience that allows individuals to be amused by their circumstances rather than being upset by them (Lefcourt et al., 1995).

Given the connection between humor and the perception of stress, it seems likely that humor may mediate the association between individual differencessuch as pathological narcissism - and stress by altering how individuals perceive and react to stressful events. One possibility is that humor may allow individuals to deal with potential threats in a way that is more positive than how they would react without a sense of humor (Kuiper, Martin, \& Olinger, 1993; Kuiper, McKenzie, \& Belanger, 1995). The transition to life as a university student is particularly interesting for our purposes because one potential cost of the pathological forms of narcissism is that they may result in individuals being particularly reactive to stress. For example, the pathological forms of narcissism have been found to be associated with negative emotions following negative events (Besser \& Priel, 2010; Besser \& Zeigler-Hill, 2010). The reactions of those with high levels of pathological narcissism may be because of these threatening events triggering underlying negative self-beliefs that may alter how these individuals 
evaluate their abilities to cope with stressful situations (Besser \& Priel, 2009; Bosson et al., 2008; Zeigler-Hill \& Jordan, in press).

Our prediction for Study 2 was that humor styles would mediate the association between the forms of pathological narcissism and perceived stress. This prediction was based on previous research demonstrating that humor styles are intimately associated with how individuals perceive and respond to stressful situations (see Lefcourt, 2002, for a review). More specifically, individuals who possess adaptive humor styles have been found to interpret potential stressors in such a way that their negative impact is limited, whereas those who possess maladaptive humor styles are often highly reactive to stressful events (Cann \& Etzel, 2008). This is important for the present study because the pathological forms of narcissism have been shown to be associated with particular humor styles such that grandiose narcissism was associated with the use of adaptive humor whereas vulnerable narcissism was associated with the use of maladaptive humor (Zeigler-Hill \& Besser, 2011). Taken together, these previous results led us to expect that the use of adaptive and maladaptive humor styles may be an important pathway by which the pathological forms of narcissism are associated with perceived stress. That is, we believed that the use of adaptive humor styles-which emphasize affiliation and self-enhancement - would foster positive relationships with others and may indirectly serve their stress-regulation goals (e.g., Leary \& Baumeister, 2000). In contrast, we expected that the use of maladaptive humor may be detrimental to stress-regulation because of the damage that it causes to interpersonal relationships. The mediational roles that we propose for adaptive and maladaptive humor styles are consistent with the results of previous studies that have examined mediational hypotheses concerning humor styles (e.g., Dozois, Martin, \& Bieling, 2009; Kazarian, Moghnie, \& Martin, 2010; Zeigler-Hill \& Besser, 2011).

\section{Method}

\section{Participants and Procedure}

Participants were 251 Jewish Israeli undergraduates (77 men and 174 women) who took part in the study during the first week of their first semester at a university or a college in the southern region of Israel. The data for Study 2 were collected at the same time and using the same basic methodology as Study 1 but different participants took part in these studies (i.e., the data are independent). As a result, the only meaningful difference between these studies was that the participants in Study 2 completed a measure of perceived stress along with measures of pathological narcissism and humor styles. The mean age of the participants was 23.56 years (range $20-30 ; S D=2.89$ ). 


\section{Measures}

The same measures from Study 1 were used to assess grandiose narcis$\operatorname{sism}(\alpha=.90)$, vulnerable narcissism $(\alpha=.89)$, affiliative humor style $(\alpha=$ $.71)$, self-enhancing humor style $\alpha=.82)$, aggressive humor style $(\alpha=.70)$, and self-defeating humor style $(\alpha=.79)$. In addition, Study 2 included a measure of perceived stress.

\section{Perceived Stress}

Perceived Stress Scale (Cohen, Kamarck, \& Mermelstein, 1983; Cohen $\&$ Williamson, 1988) is a self-report measure of perceptions of stress. The scale includes 14 items designed to measure the degree to which individuals cognitively appraise their lives as unpredictable, uncontrollable, and overwhelming. Respondents are asked to rate how often they experienced a particular feeling or thought during the past month using scales that range from 0 (never) to 4 (very often). In the present study, participants were asked to respond concerning their experiences during the transition to the university (e.g., preparations, expectations, and first week experiences). In the present sample, the Perceived Stress Scale had an internal consistency of $=.80$.

\section{Results}

\section{Data Analytic Strategy}

We examined the normality of the distributions of the variables in the present study by using the Kolmogorov-Smirnov test (K-S test; Smirnov, 1948), the Lilliefors test (Lilliefors, 1967), and the Shapiro-Wilk test (Shapiro \& Wilk, 1965). The results of these tests indicated that the distributions of these measures were relatively normal $(p s>.45)$. We also examined whether multicollinearity was a concern between pathological forms of narcissism, humor styles, and perceived stress. Eigenvalues of the scaled and uncentered cross-products matrix, condition indices, and variance decomposition proportions, along with variance inflation factors (VIF) and tolerances from multicollinearity analyses, indicated the absence of multicollinearity. SEM was used to investigate the proposed mediation model in two stages. During the first stage, SEM was used to examine the direct associations between the forms of pathological narcissism and perceived stress. Then, SEM was used to examine whether humor styles mediated the association between the pathological forms of narcissism and perceived stress following 
the basic criteria for mediation established by Baron and Kenny (1986). Although the recommendations made by Baron and Kenny (1986) are influential and have been extensively cited, some criticisms have been raised such as the use of Sobel's (1982) large sample test to evaluate the significance of indirect associations (e.g., MacKinnon, Lockwood, Hoffman, West, \& Sheets, 2002). As a result, we evaluated the proposed mediational models by studying the sampling variability of estimates of the indirect association using the bootstrap framework (Mallinckrodt, Abraham, Wei, \& Russell, 2006; Shrout \& Bolger, 2002). Using AMOS, we implemented this procedure in the direct and mediational models that involved drawing 5,000 bootstrapping samples. We found that $100 \%$ of the bootstrap samples converged for our models. The $95 \%$ confidence intervals and the confidence intervals based on the bias-corrected bootstrap for the direct and indirect associations in these models were consistent with the conclusion that the direct and indirect associations were significantly different from zero. Standard errors $(S E)$ and confidence intervals $(95 \% \mathrm{CI}$ ) based on the bias-corrected bootstrap are reported in parentheses. These results suggest that this procedure led to a stable estimate of the distributions.

\section{Direct Association Model}

The direct associations SEM model for grandiose and vulnerable narcissism had zero degrees of freedom that means that fit indices could not be estimated. As can be seen in the correlations (see Table 1), both grandiose narcissism $(r=.15, p<.02)$ and vulnerable narcissism $(r=.37, p<.001)$ were significantly associated with higher levels of perceived stress. However, the combined associations model that controlled for the shared variance between grandiose and vulnerable narcissism scales $(r=.60, p<.001)$ indicated that vulnerable narcissism had a significant unique association with perceived stress levels $[\beta=.44, t=5.93, p<.0001 ; S E=0.050,95 \% \mathrm{CI}$ $(0.18,0.38), p<.0001]$ but that grandiose narcissism did not have this sort of association $[\beta=-.11, t=-.1 .51, n s ; S E=0.06,95 \% \mathrm{CI}(-0.19,0.04)$, $n s]$. The direct association model explained $14 \%$ of the variance in perceived stress.

\section{Mediational Model}

We ran an indirect associations SEM model that included the pathological forms of narcissism, humor styles, and perceived stress. This model is depicted in Figure 3. This mediational model fit the observed 
data very well: $\chi^{2}(7)=9.41, p>.22, \chi^{2} / d f=1.35, \mathrm{NNFI}=.97, \mathrm{CFI}=$ .99 , RMSEA $=.03$ [90\% CI 0.000, 0.07]. The association between vulnerable narcissism and perceived stress was mediated $[S E=0.076$, $95 \% \mathrm{CI}(0.05,0.38), p<.001]$ by high levels of maladaptive humor $[\beta=$ $.32, t=2.81, p<.005 ; S E=0.083,95 \% \mathrm{CI}(0.04,0.36), p<.02]$ and low levels of adaptive humor $[\beta=-.43, t=-3.82, p<.0001 ; S E=$ $0.10,95 \% \mathrm{CI}(-0.49,-0.08), p<.001]$ as indicated by the findings that the direct path from vulnerable narcissism and perceived stress decreased compared to the direct associations model $[\beta=.20, t=1.86, n s ; S E=$ $0.09,95 \% \mathrm{CI}(-0.09,0.26), n s]$. Adaptive humor was associated with low levels of perceived stress $[\beta=-.34, t=-3.05, p<.002 ; S E=0.15$, 95\% CI $(-0.71,-0.12), p<.002]$ and maladaptive humor was associated with high levels of perceived stress $[\beta=.26, t=2.20, p<.03 ; S E=$ $0.19,95 \%$ CI $(0.01,0.76), p<.04]$. Comparisons of the paths were made using a z' test (MacKinnon et al., 2002) that indicated that the link between vulnerable narcissism and perceived stress was because of its association with low adaptive humor $\left(\left(\mathrm{z}^{\prime}\right)=2.43, p<.02\right)$ rather than its association with high maladaptive humor $\left(\left(\mathrm{z}^{\prime}\right)=1.80, n s\right)$. Although grandiose narcissism had no direct associations with perceived stress, as can be seen in Figure 3, it had an indirect association with low perceived stress levels $[S E=0.05,95 \% \mathrm{CI}(-0.20,-0.02), p<.02]$ through its associations with high levels of adaptive humor $[\beta=.45, t=3.94, p<$ $.0001 ; S E=0.10,95 \% \mathrm{CI}(0.11,0.51), p<.001]$. The mediating model explained $15 \%$ of the variance in adaptive humor, $17 \%$ of the variance in maladaptive humor, and $24 \%$ of the variance in perceived stress.

\section{Alternative/Competing Models}

We also performed a series of regression analyses to investigate possible competing models that included (1) whether humor styles moderated the associations between the pathological forms of narcissism and perceived stress and (2) whether perceived stress moderated the associations between the pathological forms of narcissism and humor styles. These moderating effects did not approach conventional levels of significance.

\section{Discussion}

The results of Study 2 indicate that vulnerable narcissism has a positive association with maladaptive humor styles and a negative association with 


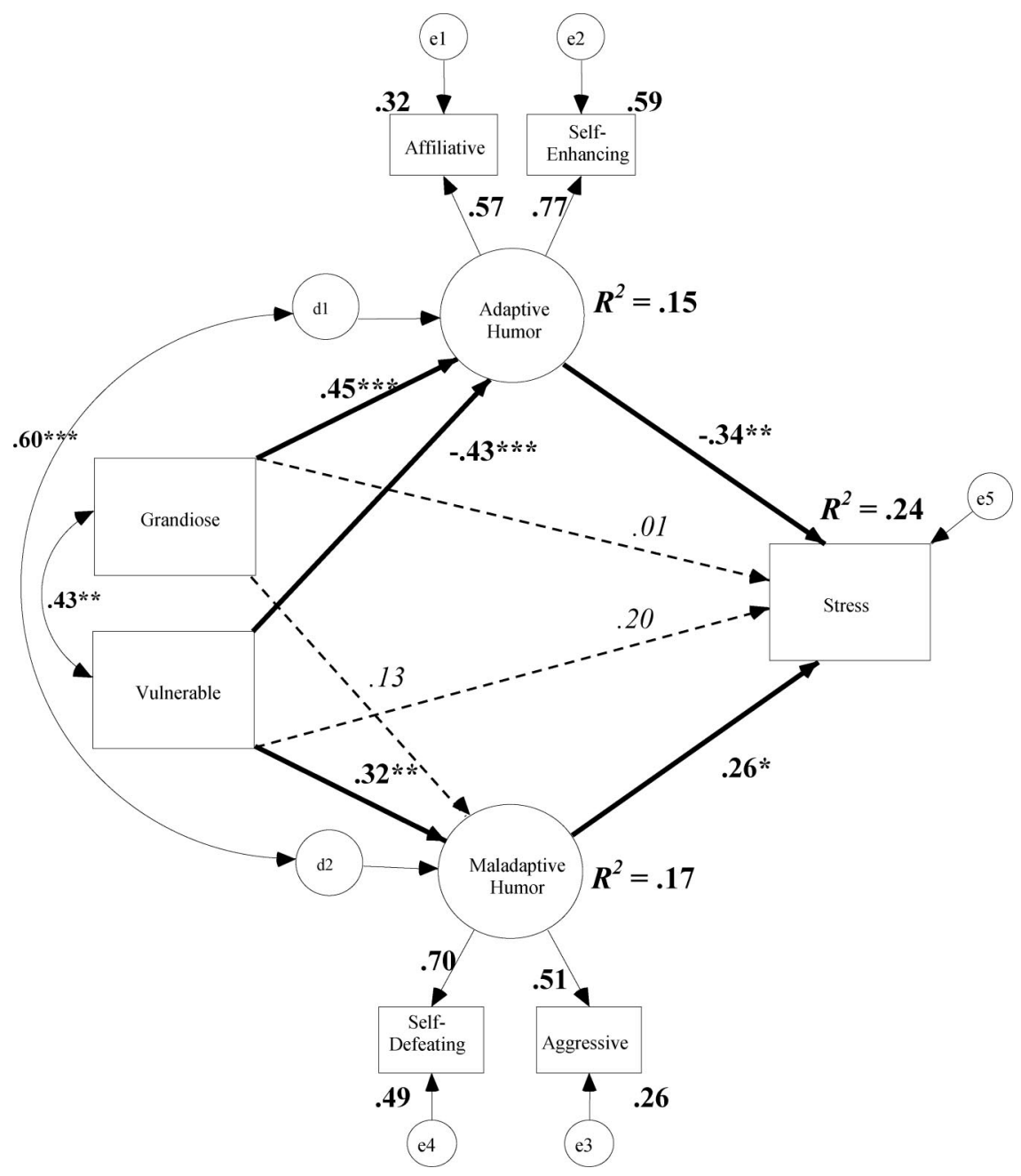

Figure 3. Study 2: Mediational model of the associations among the forms of pathological narcissism, humor styles, and perceived stress. Note. Rectangles indicate measured variables and the large circles represent latent constructs. Small circles reflect residuals (e). Bold numbers above or near endogenous variables represent the amount of variance explained $\left(R^{2}\right)$. Bidirectional arrow depicts covariance and unidirectional arrows depict hypothesized directional links. Standardized maximum likelihood parameters are used. Bold estimates are statistically significant. Wide paths indicate significant indirect/mediated association. $\Delta R^{2}=10 \%, N=251$; ${ }^{* * *} p<.01,{ }^{* * * *} p<.001$. 
adaptive humor styles, whereas grandiose narcissism is positively associated with adaptive humor styles and is not associated with the maladaptive humor styles. These results replicate the findings of Study 1. Moreover, the results of Study 2 indicated that it is vulnerable narcissism that is associated with perceived stress in the transition to the university and that the difference in humor styles used by those with high levels of vulnerable and grandiose narcissism may be a primary mechanism in these associations. That is, the perceived stress reported by individuals with high levels of vulnerable narcissism may be because of the fact that they refrain from using adaptive forms of humor. These findings are consistent with the results of previous studies showing that individual differences influence how people appraise new and threatening situations (e.g., Lazarus, 1993).

\section{GENERAL DISCUSSION}

The present studies examined the connections between pathological forms of narcissism and humor styles. Our results suggest that grandiose narcissism is associated with adaptive humor styles but not with maladaptive humor styles. That is, individuals with high levels of grandiose narcissism tend to use humor in a benign fashion that allows them to enhance both themselves and others. In contrast, vulnerable narcissism was found to have a negative association with adaptive humor styles and a positive association with maladaptive humor styles. This pattern suggests that individuals with high levels of vulnerable narcissism not only refrain from using humor in a benign way but they actually use humor in a way that causes injury both to themselves (i.e., self-defeating humor) and others (i.e., aggressive humor). The use of injurious forms of humor may stem from a misguided attempt to gain the approval and acceptance of others. These differential associations between the pathological forms of narcissism and humor styles emerged in both of the present studies. These differences in humor styles may help us better understand some of the interpersonal differences between grandiose and vulnerable narcissism that have been observed in past research (e.g., Dickinson \& Pincus, 2003).

We also examined whether humor styles mediated the association between the pathological forms of narcissism and perceived stress. Our results suggest that vulnerable narcissism is associated with perceived stress during the transition period as students become accustomed to higher education. Importantly, the association between vulnerable narcissism and perceived stress is partially explained by the fact that individuals with high levels of vulnerable narcissism tend not to use adaptive forms of humor. This suggests that vulnerable narcissism may be associated with perceived stress because 
individuals with high levels of vulnerable narcissism fail to use humor in benign ways that allow them to enhance both themselves and others.

The results of the present studies are consistent with previous findings that have shown that even though grandiose narcissism and vulnerable narcissism share certain characteristics (e.g., Dickinson \& Pincus, 2003) there are important differences between these forms of pathological narcissism (Besser \& Priel, 2010; Besser \& Zeigler-Hill, 2010; Cain et al., 2008). Grandiose narcissism may be associated with the use of adaptive forms of humor because it allows individuals with high levels of grandiose narcissism to cultivate positive relationships that they can use to maintain and enhance their tenuous feelings of self-worth. In contrast, vulnerable narcissism is associated with high levels of maladaptive humor and low levels of adaptive humor. This suggests that individuals with high levels of vulnerable narcissism tend to use injurious humor that targets either themselves or others. Injurious forms of humor may be problematic because individuals who belittle themselves may undermine how they are perceived by others and aggressive humor that is targeted at others may foster bad feelings that harm their relationships.

The finding that adaptive humor styles do not moderate the associations between the personality vulnerability factors of grandiose narcissism and vulnerable narcissism, on the one hand, and perceived stress, on the other, further suggests that even when individuals with high levels of grandiose narcissism and vulnerable narcissism use more adaptive humor styles, decreased levels of perceived stress will not be observed. However, congruent with recent theoretical assumptions (e.g., Gervais \& Wilson, 2005) and empirical findings (e.g., Besser, Luyten \& Blatt, in press; Besser, Luyten, \& Mayes, submitted for publication; Martin, 2007; Miczo, Averbeck, \& Mariani, 2009; Taber, Redden, \& Hurley, 2007; Zeigler-Hill \& Besser, 2011), the use of adaptive humor styles was associated with reduced levels of perceived stress which supports the view that humor-at least adaptive humorprotects individuals from potentially threatening events (Able, 2002; Kuiper et al., 1993, 1995; Martin et al., 1993).

\section{Clinical Implications}

The present results have a number of potentially important clinical implications. One implication is that clinicians may want to consider encouraging the use and appreciation of more adaptive styles of humor particularly among individuals with narcissistic tendencies. This is especially important because humor has been linked to several coping strategies such as distancing oneself from the stressor (Kuiper et al., 1993; Lefcourt et al., 1995), aggres- 
sive efforts toward confronting and dealing with the stress (Kuiper et al., 1993), and resolving the problems causing stress (McCrae \& Costa, 1986). Individuals who use humor tend to be more accurate and realistic in their appraisal of stress (Able, 2002; Kuiper \& Martin, 1998; Martin, 2006). It appears that individuals who lack an adaptive style of humor-such as those with high levels of vulnerable narcissism - may either be inaccurate in their appraisal of stress or more reactive to stressful events (e.g., Deaner \& McConatha, 1993). This shift toward adaptive humor could be modeled and encouraged by the clinicians during sessions to emphasize its utility in regulating stress and affect (Kruger, 1996; Lemma, 2000; Maples, Dupey, Torres-Rivera, Phan, Vereen, \& Garrett, 2001). Studies that have examined the use of humor in clinical settings have found a positive effect on treatment outcomes and the satisfaction of both clinicians and clients (e.g., Kidd, Miller, Boyd, \& Cardeña, 2009) but further research is needed to investigate the potential role of humor, specifically in the treatment of narcissistic individuals.

\section{Limitations and Directions for Future Research}

There are important limitations associated with the present studies. The first limitation is that the present studies relied exclusively on self-report measures. This leaves open the possibility that participants may not have provided accurate responses about their behaviors and perceptions. For example, some participants may have denied using injurious forms of humor even though they actually use this sort of humor. To avoid this problem, future research may want to examine humor use in controlled situations or ask the friends and family members of individuals to report on the sorts of humor that these individuals actually use in their interactions with others. The second limitation is that the only potential mediator we examined was humor styles. It is possible that other mediators may play an important role in the association between the forms of pathological narcissism and perceived stress. Moreover, the cross-sectional nature of the present research limits any assignment of causality. That is, the present studies cannot provide a definitive answer concerning the direction of the observed effects. Further research is needed to develop a clearer understanding of the causal mechanisms that link pathological narcissism, humor styles, and perceived stress. The third limitation concerns the generalizability of the present results beyond our Israeli student samples. Our concern about generalizability stems from the fact that there are cultural differences between Americans and Israelis which may impact how individuals use humor. One difference is 
that Israelis tend to have more collectivistic ideals than Americans (Oyserman, Coon, \& Kemmelmeier, 2002) that may lead to greater use of benign humor styles. Another difference is that American students often make the transition to college soon after completing high school whereas Israeli youth are required to complete 2-4 years of compulsory military service before they are able to attend university. As a result, most Israeli students who enter college have already had some experience being away from home and adjusting to a new social environment that may provide a sort of buffer to the stress associated with the transition to college (see Breznitz \& Eshel, 1983, for a review). Despite their relative maturity and life experience compared to American students, it is important to note that Israeli students report considerable levels of stress during the transition to college and face their own stressors (e.g., more time has passed since they graduated from high school). These cultural differences suggest that future research should attempt to replicate the present findings in a culture with less emphasis on collectivistic qualities and in which students usually enter college immediately after high school.

\section{CONCLUSION}

The results of the present studies suggest that the way individuals feel about themselves tends to influence how they use humor. More specifically, grandiose narcissism was associated with the use of self-enhancing humor and affiliative humor. In contrast, vulnerable narcissism was associated with the use of aggressive and self-defeating styles of humor as well as refraining from the use of self-enhancing and affiliative styles of humor. Further, adaptive humor was found to mediate the association between vulnerable narcissism and perceived stress such that low levels of adaptive humor may explain the elevated level of perceived stress reported by those with high levels of vulnerable narcissism. These findings may have implications for the link between personality vulnerabilities and stress as well as for the role of humor in clinical interventions.

\section{REFERENCES}

Abel, M. H. (1998). Interaction of humor and gender in moderating relationships between stress and outcomes. Journal of Psychology: Interdisciplinary and Applied, 132, 267-276.

Abel, M. H. (2002). Humor, stress, and coping strategies. Humor: International Journal of Humor Research, 15, 365-381.

Allport, G. W. (1961). Pattern and growth in personality. New York: Holt, Rinehart \& Winston. 
American Psychiatric Association. (2000). Diagnostic and statistical manual of mental disorders (4th ed., Text revision). Washington, DC: Author.

Anderson, J. C., \& Gerbing, D. W. (1988). Structural equation modeling in practice: A review and recommended two-step approach. Psychological Bulletin, 103, 411-423.

Arbuckle, J. L. (2009). Amos 18 user's guide. Crawfordville, FL: Amos Development Corporation.

Baron, R. M., \& Kenny, D. A. (1986). The moderator-mediator variable distinction in social psychological research: Conceptual, strategic, and statistical considerations. Journal of Personality and Social Psychology, 51, 1173-1182.

Besser, A., Luyten, P., \& Blatt, S. J. (in press). Do humor styles mediate or moderate the relationship between self-criticism and neediness, and depressive symptoms? Journal of Nervous and Mental Disease.

Besser, A., Luyten, P., \& Mayes, L. C. Adult attachment and distress: The mediating role of humor styles. Manuscript submitted for publication.

Besser, A., \& Priel, B. (2009). Emotional responses to a romantic partner's imaginary rejection: The roles of attachment anxiety, covert narcissism, and self-evaluation. Journal of Personality, 77, 287-325.

Besser, A., \& Priel, B. (2010). Grandiose narcissism versus vulnerable narcissism in threatening situations: Emotional reactions to achievement failure and interpersonal rejection. Journal of Social and Clinical Psychology, 29, 874-902.

Besser, A., \& Zeigler-Hill, V. (2010). The influence of pathological narcissism on emotional and motivational responses to negative events: The roles of visibility and concern about humiliation. Journal of Research in Personality, 44, 520-534.

Bosson, J. K., Lakey, C. E., Campbell, W. K., Zeigler-Hill, V., Jordan, C. H., \& Kernis, M. H. (2008). Untangling the links between narcissism and self-esteem: A theoretical and empirical review. Social and Personality Psychology Compass, 2, 1415-1439.

Breznitz, S., \& Eshel, Y. (1983). Life events: Stressful ordeal or valuable experience. In S. Breznitz (Ed.), Stress in Israel (pp. 228-261). New York: Van Nostrand Reinhold Co.

Cain, N. M., Pincus, A. L., \& Ansell, E. B. (2008). Narcissism at the crossroads: Phenotypic description of pathological narcissism across clinical theory, social/personality psychology, and psychiatric diagnosis. Clinical Psychology Review, 28, 638-656.

Cann, A., \& Etzel, K. C. (2008). Remembering and anticipating stressors: Positive personality mediates the relationship with sense of humor. Humor: International Journal of Humor Research, 21, 157-178.

Cohen, S., Kamarck, T., \& Mermelstein, R. (1983). A global measure of perceived stress. Journal of Health and Social Behavior, 24, 386-396.

Cohen, S., \& Williamson, G. (1988). Perceived stress in a probability sample of the United States. In S. Spacapan \& S. Oskamp (Eds.), The social psychology of health (pp. 31-67). Newbury Park, CA: Sage.

Cooper, A. M. (1998). Further developments in the clinical diagnosis of narcissistic personality disorder. In E. Ronningstam (Ed.), Disorders of narcissism: Diagnostic, clinical, and empirical implications (pp. 53-74). Washington, DC: American Psychiatric Press.

Deaner, S. L., \& McConatha, J. T. (1993). The relationship of humor to depression and personality. Psychological Reports, 72, 755-763.

Dickinson, K. A., \& Pincus, A. L. (2003). Interpersonal analysis of grandiose and vulnerable narcissism. Journal of Personality Disorders, 17, 188-207.

Dozois, D. J. A., Martin, R. A., \& Bieling, P. J. (2009). Early maladaptive schemas and adaptive/maladaptive styles of humor. Cognitive Therapy and Research, 33, 585-596.

D'Zurilla, T. J., \& Sheedy, C. F. (1991). Relation between social problem-solving ability and subsequent level of psychological stress in college students. Journal of Personality and Social Psychology, 61, 841-846.

Edelstein, R. S., Yim, I. S., \& Quas, J. A. (2010). Narcissism predicts heightened cortisol 
reactivity to a psychosocial stressor in men. Journal of Research in Personality, 44, $565-572$.

Foa, U. G., \& Foa, E. B. (1974). Societal structures of the mind. Springfield, IL: Charles C Thomas.

Fram, E. H., \& Bonvillian, G. (2001). Employees as part-time students: Is stress threatening the quality of their business education? Advanced Management Journal, 66, 30-35.

Freud, S. (1905/1960). Jokes and their relation to the unconscious (J. Strachey, Trans.). New York: Norton. (Original work published 1905)

Freud, S. (1928). Humour. International Journal of Psychoanalysis, 9, 1-6.

Gabbard, G. O. (1989). Two subtypes of the narcissistic personality disorder. Bulletin of the Menninger Clinic, 53, 527-532.

Gabbard, G. O. (1998). Transference and countertransference in the treatment of narcissistic patients. In E. Ronningstam (Ed.), Disorders of narcissism: Diagnostic, clinical, and empirical implications (pp. 53-74). Washington, DC: American Psychiatric Press.

Galloway, G. (2010). Individual differences in personal humor styles: Identification of prominent patterns and their associates. Personality and Individual Differences, 48, 563-567.

Gersten, S. P. (1991). Narcissistic personality disorder consists of two distinct subtypes. Psychiatric Times, 8, 25-26.

Gervais, M., \& Wilson, D. S. (2005). The evolution and function of laughter and humor: A synthetic approach. Quarterly Review of Biology, 80, 395-430.

Gurtman, M. B. (1992). Construct validity of interpersonal personality measures: The interpersonal circumplex as a nomological net. Journal of Personality and Social Psychology, 63, 105-118.

Haggis, T., \& Pouget, M. (2002). Trying to be motivated: Perspectives on learning from younger students accessing higher education. Teaching in Higher Education, 7, 323-336.

Hoyle, R. H., \& Smith, G. T. (1994). Formulating clinical research hypotheses as structural equation models: A conceptual overview. Journal of Consulting and Clinical Psychology, 62, 429-440.

Hu, L. T., \& Bentler, P. M. (1999). Cutoff criteria for fit indexes in covariance structure analysis: Conventional criteria versus new alternatives. Structural Equation Modeling, 6, $1-55$.

Kazarian, S. S., Martin, R. A. (2004). Humor styles, personality, and well-being among Lebanese university students. European Journal of Personality, 18, 209-219.

Kelloway, E. K. (1998). Using LISREL for structural equation modeling: A researcher's guide. Newbury Park, CA: Sage.

Kelsey, R. M., Ornduff, S. R., McCann, C. M., \& Reiff, S. (2001). Psychophysiological characteristics of narcissism during active and passive coping. Psychophysiology, 38, 292-303.

Kidd, S. A., Miller, R., Boyd, G. M., \& Cardeña, I. (2009). Relationships between humor, subversion, and genuine connection among persons with severe mental illness. Qualitative Health Research, 19, 1421-1430.

Kruger, A. (1996). The nature of humor in human nature: Cross-cultural commonalities. Counseling Psychology Quarterly, 8, 235-242.

Kuiper, N. A., \& Martin, R. A. (1998). Is sense of humor a positive personality characteristic? In W. Ruch (Ed.), The sense of humor: Explorations of a personality characteristic (pp. 159-178). Berlin, Germany: Walter de Gruyter.

Kuiper, N. A., Martin, R. A., \& Olinger, L. J. (1993). Coping humour, stress, and cognitive appraisals. Canadian Journal of Behavioural Science, 25, 81-96.

Kuiper, N. A., McKenzie, S. D., \& Belanger, K. A. (1995). Cognitive appraisals and individual differences in sense of humor: Motivational and affective implications. Personality and Individual Differences, 19, 359-372.

Labott, S. M., Ahleman, S., Wolever, M. E., \& Martin, R. B. (1990). The physiological and 
psychological effects of the expression and inhibition of emotion. Behavioral Medicine, 16, 182-189.

Lazarus, R. S. (1993). Coping theory and research: Past, present, and future. Psychosomatic Medicine, 55, 234-247.

Lazarus, R. S., \& Folkman, S. (1984). Stress, appraisal, and coping. New York: Springer.

Leary, M. R., \& Baumeister, R. F. (2000). The nature and function of self-esteem: Sociometer theory. In M. P. Zanna (Ed.), Advances in experimental social psychology (Vol. 32; pp. 1-62). San Diego, CA: Academic Press.

Lefcourt, H. M. (2002), Humor. In C. R. Snyder \& S. J. Lopez (Eds.), Handbook of positive psychology (619-631). New York: Oxford University Press.

Lefcourt, H. M., Davidson, K., Shepherd, R., Phillips, M., Prkachin, K. M., \& Mills, D. E. (1995). Perspective-taking humor: accounting for stress moderation. Journal of Social and Clinical Psychology, 14, 373-391.

Lemma, A. (2000). Humour on the couch: Exploring humour in psychotherapy and everyday life. Philadelphia: Whurr.

Lilliefors, H. (1967). On the Kolmogorov-Smirnov test for normality with mean and variance unknown. Journal of the American Statistical Association, 62, 399-402.

Macan, T. H., Shahani, C., Dipboye, R. L., \& Phillips, A. P. (1990). College students' time management: Correlations with academic performance and stress. Journal of Educational Psychology, 82, 760-768.

MacKinnon, D. P., Lockwood, C. M., Hoffman, J. M., West, S. G., \& Sheets, V. (2002). A comparison of methods to test mediation and other intervening variable effects. Psychological Methods, 7, 83-104.

Malach-Pines, A., \& Keinan, G. (2007). Stress and burnout in Israeli police officers during a Palestinian uprising (Intifada). International Journal of Stress Management, 14, 160-174.

Mallinckrodt, B., Abraham, T. W., Wei, M., \& Russell, D. W. (2006). Advances in testing statistical significance of mediation effects. Journal of Counseling Psychology, 53, 372378.

Maples, M. F., Dupey, P., Torres-Rivera, E., Phan, L. T., Vereen, L., \& Garrett, M. T. (2001). Ethnic diversity and the use of humor in counseling: Appropriate or inappropriate? Journal of Counseling \& Development, 79, 53-60.

Martin, R. A. (2006). The Situational Humor Response Questionnaire (SHRQ) and Coping Humor Scale (CHS): A decade of research findings. Humor: International Journal of Humor Research, 9, 251-272.

Martin, R. A. (2007). The psychology of humor: An integrative approach. Burlington, MA: Elsevier.

Martin, R. A., Kuiper, N. A., Olinger, L. J., \& Dance, K. A. (1993). Humor, coping with stress, self-concept, and psychological well-being. Humor: International Journal of Humor Research, 6, 89-104.

Martin, R. A., Puhlik-Doris, P., Larsen, G., Gray, J., \& Weir, K. (2003). Individual differences in uses of humor and their relation to psychological well-being: Development of the Humor Styles Questionnaire. Journal of Research in Personality, 37, 48-75.

Maslow, A. H. (1954). Motivation and personality. New York: Harper \& Row.

McCrae, R. R., \& Costa, P. T. (1986). Personality, coping, and coping effectiveness in an adult sample. Journal of Personality, 54, 385-405.

Miczo, N., Averbeck, J. M., \& Mariani, T. (2009). Affiliative and aggressive humor, attachment dimensions, and interaction goals. Communication Studies, 60, 443-459.

Miller, J. D., \& Campbell, W. K. (2008). Comparing clinical and social-personality conceptualizations of narcissism. Journal of Personality, 76, 449-476.

Morf, C. C., \& Rhodewalt, F. (2001). Unraveling the paradoxes of narcissism: A dynamic self-regulatory processing model. Psychological Inquiry, 12, 177-196.

Oyserman, D., Coon, H. M., \& Kemmelmeier, M. (2002). Rethinking individualism and 
collectivism: Evaluation of theoretical assumptions and meta-analyses. Psychological Bulletin, 128, 3-72.

Pincus, A. L., Ansell, E. B., Pimentel, C. A., Cain, N. M., Wright, A. G. C., \& Levy, K. N. (2009). Initial construction and validation of the Pathological Narcissism Inventory. Psychological Assessment, 21, 365-379.

Pincus, A. L., \& Lukowitsky, M. R. (2010). Pathological narcissism and narcissistic personality disorder. Annual Review of Clinical Psychology, 6, 421-446.

Raskin, R. N., \& Hall, C. S. (1979). A narcissistic personality inventory. Psychological Reports, 45, 590.

Raskin, R. N., \& Hall, C. S. (1981). The Narcissistic Personality Inventory: Alternate form reliability and further evidence of construct validity. Journal of Personality Assessment, 45, 159-162.

Ruiz, J. M., Smith, T. W., \& Rhodewalt, F. (2001). Distinguishing narcissism and hostility: Similarities and differences in interpersonal circumplex and five-factor correlates. Journal of Personality Assessment, 76, 537-555.

Shapiro, S. S., \& Wilk, M. B. (1965). An analysis of variance test for normality. Biometrika, 52, 591-611.

Shirom, A. (1986). On the cross-environment generality of the relational view of stress. Journal of Environmental Psychology, 6, 121-134.

Shrout, P. E., \& Bolger, N. (2002). Mediation in experimental and nonexperimental studies: New procedures and recommendations. Psychological Methods, 7, 422-445.

Smirnov, N. V. (1948). Tables for estimating the goodness of fit of empirical distributions. Annals of Mathematical Statistics, 19, 279.

Sobel, M. E. (1982). Asymptotic confidence intervals for indirect effects in structural equation models. In S. Leinhardt (Ed.), Sociological methodology (pp. 290-312). American Sociological Association: Washington, DC.

Sommer, K. L., Kirkland, K. L., Newman, S. R., Estrella, P., \& Andreassi, J. L. (2009). Narcissism and cardiovascular reactivity to rejection imagery. Journal of Applied Social Psychology, 39, 1083-1115.

Taber, K. H., Redden, M., \& Hurley, R. A. (2007). Functional anatomy of humor: Positive affect and chronic mental illness. Journal of Neuropsychiatry and Clinical Neurosciences, 19, $358-362$.

Towbes, L. C., \& Cohen, L. H. (1996). Chronic stress in the lives of college students: Scale development and prospective prediction of distress. Journal of Youth and Adolescence, 25, 199-217.

Tritt, S., Ryder, A. G., Ring, A., \& Pincus, A. L. (2010). Pathological narcissism and the depressive temperament. Journal of Affective Disorders, 122, 280-284.

Trueman, M., \& Hartley, J. (1996). A comparison between the time management skills and academic performance of mature and traditional entry university students. Higher Education, 32, 199-215.

Vermunt, R., \& Steensma, H. (2005). How can justice be used to manage stress in organizations? In J. Greenberg \& J. A. Colquitt (Eds.), Handbook of organizational justice (pp. 383-410). Mahwah, NJ: Erlbaum.

Vernon, P. A., Martin, R. A., Schermer, J. A., \& Mackie, A. (2008). A behavioral genetic investigation of humor styles and their correlations with the Big-5 personality dimensions. Personality and Individual Differences, 44, 1116-1125.

Veselka, L., Schermer, J. A., Martin, R. A., \& Vernon, P. A. (2010). Relations between humor styles and the Dark Triad traits of personality. Personality and Individual Differences, 48 , 772-774.

Walker, L., Matthew, B., \& Black, F. (2004). Widening access and student non-completion: An inevitable link? Evaluating the effects of the Top-Up Programme on student completion. International Journal of Lifelong Education, 23, 43-59. 
Wink, P. (1991). Two faces of narcissism. Journal of Personality and Social Psychology, 61, 590-597.

Wright, A. G. C., Lukowitsky, M. R., Pincus, A. L., \& Conroy, D. E. (2010). The higher order factor structure and gender invariance of the Pathological Narcissism Inventory. Assessment, 17, 467-483.

Zeigler-Hill, V. (2010). The interpersonal nature of self-esteem: Do different measures of self-esteem possess similar interpersonal content? Journal of Research in Personality, 44, 22-30.

Zeigler-Hill, V., \& Besser, A. (2011). Humor style mediates the association between pathological narcissism and self-esteem. Personality and Individual Differences, 8, 1196-1201.

Zeigler-Hill, V., Clark, C. B., \& Beckman, T. E. (in press). Fragile self-esteem and the interpersonal circumplex: Are feelings of self-worth associated with interpersonal style? Self and Identity.

Zeigler-Hill, V., Clark, C. B., \& Pickard, J. D. (2008). Narcissistic subtypes and contingent self-esteem: Do all narcissists base their self-esteem on the same domains? Journal of Personality, 76, 753-774.

Zeigler-Hill, V., \& Jordan, C. H. (in press). Behind the mask: Narcissism and implicit self-esteem. In W. K. Campbell \& J. Miller (Eds.), Handbook of narcissism and narcissistic personality disorder: Theoretical approaches, empirical findings, and treatment. Hoboken, NJ: Wiley.

\section{New Journal Announcement: Psychology of Popular Media Culture}

The Publications and Communications Board of the American Psychological Association has announced that it will begin publishing the journal Psychology of Popular Media Culture in 2012. Psychology of Popular Media Culture, to be published quarterly, will be a scholarly journal dedicated to publishing empirical research and papers on how popular culture and general media influence individual, group, and system behavior.

The journal will solicit rigorous research studies, as well as data-driven theoretical papers on constructs, consequences, program evaluations, and trends related to popular culture and various media sources. Although the journal welcomes and encourages submissions from a wide variety of disciplines, topics should be linked to psychological theory and research.

The journal is accepting electronic submissions via the journal's Manuscript Submission Portal under the Instructions to Authors at http://www.apa.org/ pubs/journals/ppm. 